\title{
Orientation-contingent color aftereffects mediated by subjective transparent structures
}

\author{
TAKEO WATANABE, GEORGE L. ZIMMERMAN, and PATRICK CAVANAGH \\ Harvard University, Cambridge, Massachusetts
}

\begin{abstract}
We examined whether the orientation-contingent color aftereffect (the McCollough effect) could be mediated by subjective horizontal and vertical structure induced by the perception of transparency. In our experiments, red vertical bars and green horizontal bars were alternated as an adapting stimulus. After adaptation, subjects $(n=6)$ were asked to adjust the green and red saturation of a test pattern until they obtained a neutral gray. Horizontal and vertical stripes were combined in the test pattern in three different ways: (1) overlapping with a luminance combination that gave rise to a perception of transparent overlays of horizontal and vertical stripes (valid transparency condition), (2) overlapping with luminance combinations that did not induce a perception of transparency (invalid transparency condition) and that appeared more as a patchwork of checks, and (3) presented in adjacent, nonoverlapping areas. Our results showed that the McCollough effect was significantly greater in the valid transparency condition than in the invalid transparency conditions. The effect in the valid transparency condition was nevertheless less strong than was the effect seen with the standard test stimulus made up of nonoverlapping vertical and horizontal stripes. Our results suggest that the McCollough effect can be mediated by the subjective spatial organization (inner representation of vertical and horizontal stripes) that accompanies the perception of transparency in our stimulus.
\end{abstract}

When two patterns overlap, they produce a third region that can be seen either as a region bounded by L-shaped areas (Figure 1A) or as belonging to both patterns (Figure $1 B$ ). In the second case, one square appears to lie in front of the other. This phenomenon is called subjective transparency (e.g., Beck, Prazdny, \& Ivry, 1984; for a review, see Kersten, 1991), and it indicates the ability of the visual system to attribute more than one surface quality, in particular two depths (near and far surfaces), to a single point on the retinal image.

Beck et al. (1984) and Metelli (1974) have shown that observers report an impression of overlapping transparent surfaces in a flat image like Figure $1 \mathrm{~B}$ as long as the light values in the image correspond to the following two simple constraints derived from the physical rules of transparency: (1) the direction of lightness difference across the border of an object does not change if it is covered by a transparent sheet, but (2) the lightness difference itself must be reduced.

A two-dimensional stimulus like that in Figure 1A has several potential three-dimensional organizations. When the luminances in the figure are appropriate for transparency, subjective grouping occurs that links regions together into larger forms. For example, Watanabe and Cavanagh (1991, in press) have shown that overlapping

This work was supported by AFOSR Grant 91-0169 to P.C. G.L.Z.'s present address is Department of Electrical Engineering, Tulane University, New Orleans, Louisiana. We thank Charles F. Stromeyer III for his valuable comments on our study. Correspondence concerning this article should be addressed to T. Watanabe, Department of Psychology, Harvard University, 33 Kirkland Street, Cambridge, MA 02138. digits are easier to recognize when they appear transparent. At luminance values where the digits do not appear transparent, the digits appear more as a jumble of fragments.

In the present study, we report that the subjective grouping accompanying the perception of transparency is also relevant for the orientation-contingent color aftereffect originally studied by McCollough (1965). This suggests that the internal representation of stimuli that induce transparency can influence not only higher levels of visual processing, such as recognition (de Weert, 1986; Watanabe \& Cavanagh, 1991, in press), but also very early levels, for there is a considerable evidence that the McCollough effect occurs at the earliest stage of visual cortical processing, as will be discussed in more detail later.

In the paradigm of the McCollough effect, an observer views a red-and-black vertically striped pattern alternating every few seconds with a green-and-black horizontally striped pattern. If after several minutes of adaptation, the observer views a test stimulus made up of white and black vertical stripes, it appears to be tinged in green, whereas a test stimulus of white and black horizontal stripes appears to be tinged in red. That is, the stripes appear in a color complementary to the color of the adaptation stripes that have the same orientation. The $\mathrm{McCol}-$ lough effect is based on orientation, suggesting that its site must at least be cortical where the first orientationspecific units arise (Dodwell \& O'Shea, 1987; Livingstone \& Hubel, 1987; Stromeyer, 1978). However, the effect is also largely monocular (MacKay \& MacKay, 1975; McCollough, 1965; Murch, 1972; Skowbo, Timmney, Gentry, \& Morant, 1975; Stromeyer, Lange, \& 

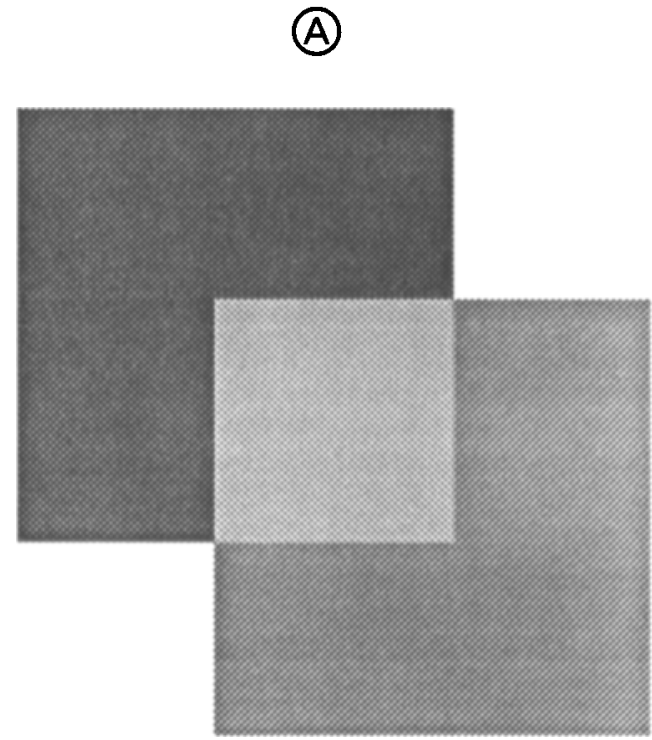

(B)

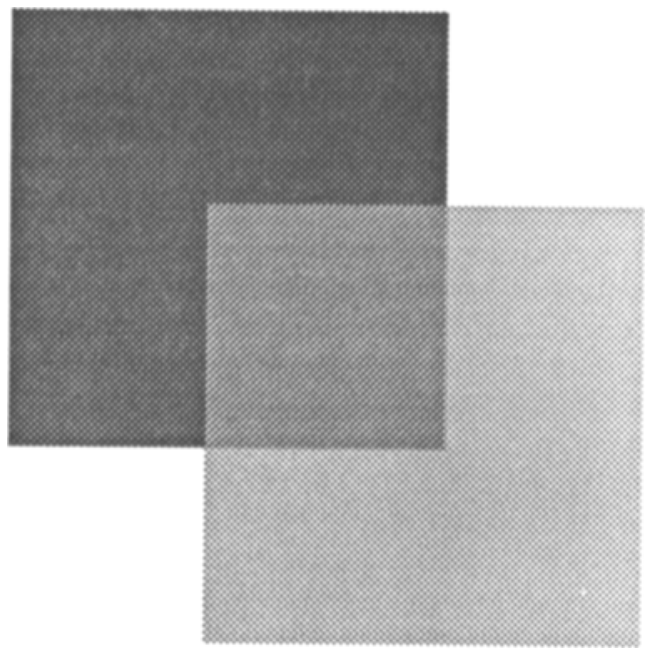

Figure 1. Stimuli in which the luminance combination is $(A)$ not valid for transparency and (B) valid for transparency.

Ganz, 1973; White \& Riggs, 1975), suggesting a site that precedes the emergence of binocular units and that also occurs in the first area of the visual cortex (Hubel \& Wiesel, 1962). These two results are somewhat conflicting, but they imply that the locus of the McCollough effect is probably in the earliest stages of cortical visual processing. Actually, cells with response properties consistent with the McCollough effect have been found in area V1 (Dow and Gouras, 1973). These cells are conjointly selective to form, color, and orientation, respond well to achromatic patterns such as white test patterns, and are largely monocular (see Stromeyer, 1978).

In our experiment, we used a patchwork checkerboard (Figure 2) in which an overlapping vertical and horizontal structure is the most salient when the luminance conditions are valid for transparency. The subjective organization of this stimulus is strongly affected by the luminance of the intersection squares common to both the horizontal and vertical stripes. When the intersection luminance simulates physical transparency, overlapping vertical and horizontal gratings are seen. When the intersection luminance does not support transparency, the horizontal and vertical stripes become less salient and a patchwork of square checks becomes evident. We wished to examine whether an orientation-contingent effect, the McCollough effect, would be influenced by the horizontal and vertical structure that is revealed in this stimulus when transparency is seen. Such an observation would demonstrate that high-level factors such as transparency can influence processing in early vision.

\section{METHOD}

\section{Subjects}

The subjects were 3 males and 3 females between the ages of 25 and 43 years. All of them were naive for this experiment. They all had normal or corrected-to-normal visual acuity (Snellen 20/20) and normal color vision (no errors in the Ishihara test).

\section{Materials}

The stimuli were presented on a color video display (Apple M0401, $640 \times 480$ pixel resolution) controlled by a Macintosh II cx computer and viewed at a distance of $57.3 \mathrm{~cm}$. The width and height of the display were $23^{\circ} \times 17.25^{\circ}$. The computer also recorded the subjects' responses.

The two adapting stimuli consisted of square wave gratings of 2 cycles per degree that covered the whole screen. In one adapting stimulus, a vertical grating was presented that consisted of alternating red and black stripes. The CIE $x, y$ chromaticity coordinates of the red were $0.63,0.33$, and its luminance was $28.5 \mathrm{~cd} / \mathrm{m}^{2}$, as measured by a Minolta Chroma Meter II. Luminance of the black regions was $0.5 \mathrm{~cd} / \mathrm{m}^{2}$. In the other adapting stimulus, a horizontal grating was presented that consisted of alternating green and black stripes. The CIE coordinates of the green were $0.28,0.59$, and the luminance was $77.0 \mathrm{~cd} / \mathrm{m}^{2}$.

Four test patterns were used, as shown in Figure 2. Figure 2A is called the valid transparency pattern because the vertical and horizontal stripes appear to overlay each other. The luminance of the nonoverlapping portions of the vertical and horizontal bars wis $50.0 \mathrm{~cd} / \mathrm{m}^{2}$. The luminances of the intersections and the background were 75.0 and $0.5 \mathrm{~cd} / \mathrm{m}^{2}$, respectively. This luminance combination was valid for Metelli's (1974) law mentioned in the introduction. Figures $2 \mathrm{~B}$ and $2 \mathrm{C}$ are the same except that the luminances of the intersection square are $25 \mathrm{~cd} / \mathrm{m}^{2}$ and $125 \mathrm{~cd} / \mathrm{m}^{2}$, respectively. These figures are called the invalid transparency patterns, because the luminances of intersection were too low for Metelli's law in Figure 2B and too high in Figure 2C. In Figure 2D, two vertical and two horizontal gratings (each of which consists of bright stripes on the dark background) are presented in physically separate areas with no overlap. The luminances of the bars and the background were the same as those in other figures. These four test patterns had the same spatial frequency as the adapting stimuli.

The subjects could alter the color appearance of the nonoverlapping sections of the bars (those with luminance of $50.0 \mathrm{~cd} / \mathrm{m}^{2}$ ) by moving a mouse connected to the computer. In all the conditions, moving the mouse leftward caused the vertical elements of the pattern to shift their hue towards green and the horizontal elements to shift towards red. Moving the mouse rightward caused the 


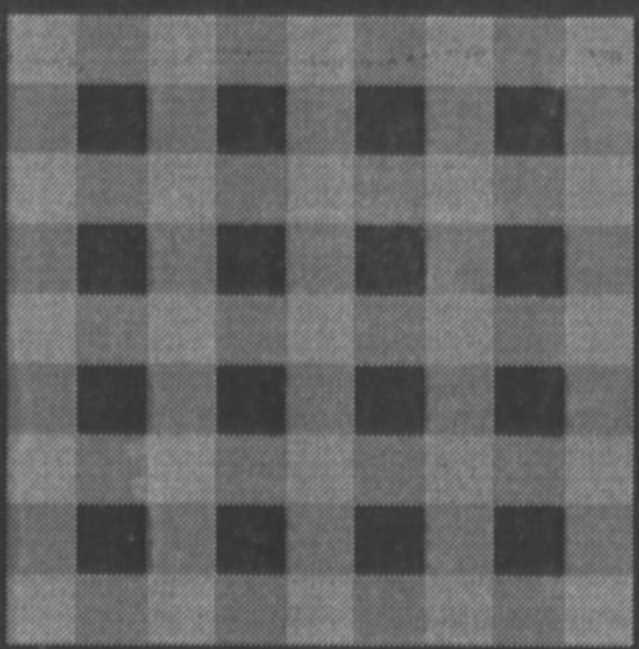

(a)

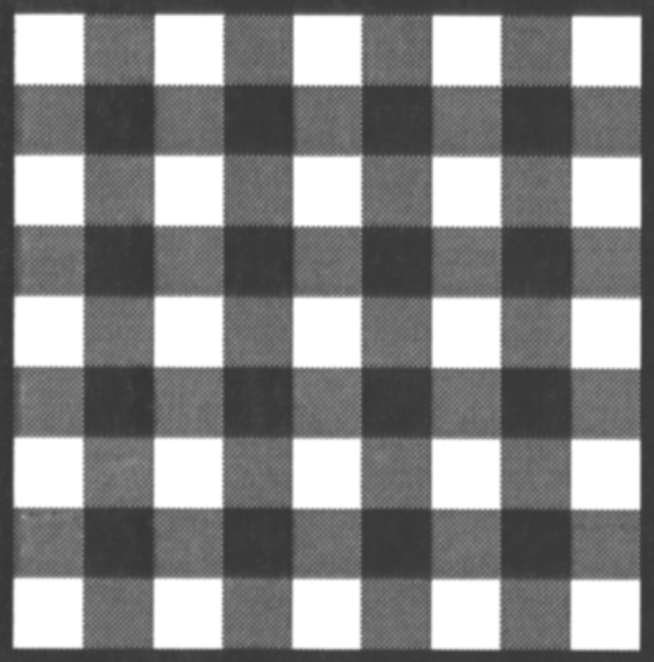

(c)

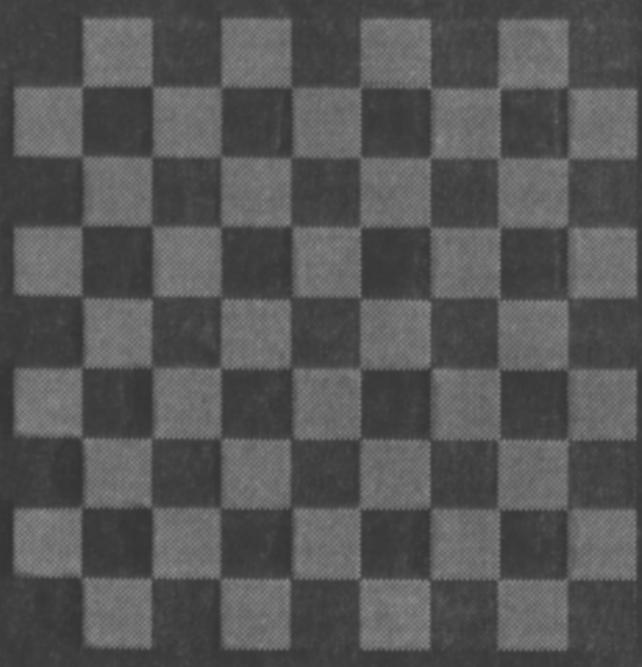

(b)
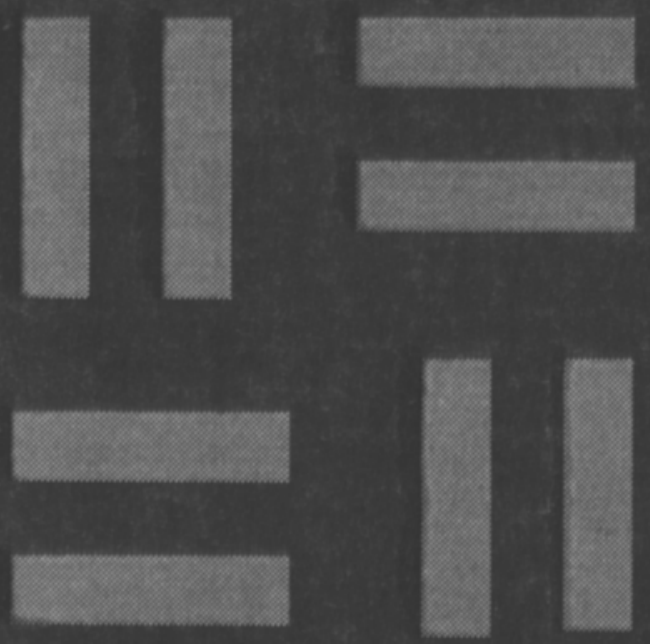

(d)

Figure 2. Depiction of the test figures. The intersections were (A) valid, (B) too dark, and (C) too bright for transparency. In (D), vertical and horizontal stripes were physically separated. These figures demonstrate the differences among the test figures but are not exact reproductions of them (see Method for detailed descriptions). 
nonoverlapping, vertical sections of the pattern to change gradually from white to red and the nonoverlapping, horizontal sections to change from white to green. At an intermediate setting, both horizontal and vertical elements were neutral gray for all conditions. The outputs of the R, G, B guns of the monitor were yoked together in software so that saturation changed with mouse displacement while luminance remained constant. The CIE $x, y$ chromaticity coordinates of the vertical stripes were $0.31,0.42$ at the rightmost position of the mouse, $0.33,0.33$, at the center, and $0.47,0.33$ at the leftmost position. The chromaticity of horizontal stripes was $0.47,0.33$ at the rightmost position of the mouse, $0.33,0.33$ at the center, and $0.31,0.42$ at the leftmost position.

\section{Procedure}

Initially, the subjects were exposed to the two adapting stimuli, vertical red stripes and horizontal green stripes, alternating at 4sec intervals. The total inspection time was $15 \mathrm{~min}$. To prevent the systematic buildup of negative afterimages, the subjects were instructed not to fixate any particular region of the adapting stimuli, but rather to let their gaze wander around the colored gratings. Immediately after adaptation, testing began. At first, the test pattern whose intersections were too dark for transparency (Figure 2B) was presented. The initial red/green saturation of the vertical and horizontal elements was determined randomly. The subjects' task was to adjust the saturation of the test pattern until the hues of the nonoverlapping portions of the horizontal and vertical bar segments matched (color cancellation technique; e.g., Riggs, White, \& Eimas, 1974; Webster, Crassini, \& Willenberg, 1987). The amount of saturation necessary to make this match was expressed as the mean excitation purity (Hurvich, 1981) of the horizontal and vertical segments. The excitation purity of each segment was defined as the ratio of the distance between the display white point $(0.33$ and 0.33 in the CIE $x, y$ chromaticity coordinates) and the CIE chromaticity coordinates of the color to the distance of the dominant wavelength of the color from the white point (i.e., extrapolating the line from white through the chromaticity coordinates for the horizontal or vertical segment to the spectrum locus). The mean excitation purity was the average of the excitation purities for the horizontal and vertical segments. These two values increased in tandem in the two segments under control of the subjects. The mean excitation purity has a value of 0.0 when both segments are set to white and 0.37 at the maximum setting available to the subjects. It has a maximum value of 0.77 in the display when the segments are red and green (red phosphor and green phosphor alone in each). If the subjects saw green in the vertical structure and red in the horizontal structure in a test stimulus and added red in the vertical structure and green in the horizontal structure, plus was assigned to the measured excitation purity. If the subjects saw red in the vertical structure and green in the horizontal structure in a test stimulus and added green in the vertical structure and red in the horizontal structure, minus was assigned to the measured excitation purity. Immediately after the subject clicked the mouse to indicate an acceptable setting, the adapting stimuli were presented again for $24 \mathrm{sec}$ (the two adaptation stimuli alternating in 4-sec intervals). A new test pattern was then presented (the valid transparency pattern, Figure $2 \mathrm{~A}$ ) and the setting procedure repeated. The same sequence of adaptation, followed by test, continued for the remaining 2 test patterns (grid with intersections too bright for transparency, Figure $2 \mathrm{C}$, and the test with physically separated horizontal and vertical gratings, Figure 2D), and the 4-test cycle continued for a total of 20 repetitions. Thus, the whole experimental session consisted of 80 settings ( 4 test patterns and 20 repetitions) and lasted about $90 \mathrm{~min}$.

To determine the initial baseline settings, the subjects performed the cancellation task for each of the four test stimuli in Figure 2 prior to the adaptation sessions.

To establish the degree of transparency visible in the stimuli, each subject was shown the valid and invalid (too-dark and too-bright) transparent test patterns and asked, "Do you see grids of horizontal and vertical stripes, one of which appears to be transparent, lying over the other?" All 6 subjects answered "yes" with the valid transparent pattern (Figure 2B), but none did so for either of the two patterns with luminances that were invalid for transparency (Figures $2 \mathrm{~A}$ and $2 \mathrm{C}$ ). ${ }^{1}$ The valid transparent pattern has two possible perceptual organizations depending on whether the vertical or the horizontal stripes look transparent (and in front). However, the subjects reported that whichever ordering they saw initially, it remained constant during each setting, which usually lasted less than $10 \mathrm{sec}$.

\section{RESULTS}

The mean saturation of red and green (expressed as excitation purity) added to the test stimulus to cancel the apparent colors induced by adaptation in the experiment was taken as a measure of the strength of adaptation.

Figure 3 shows mean saturation needed to cancel the apparent colors for the invalid (too-dark) test pattern, valid test pattern, invalid (too-bright) test pattern, and physically separated test pattern. Individual data are consistent in that the saturation is higher for the valid luminance combination than for the too-dark and too-bright luminance combinations. For 5 of the 6 subjects, the saturation needed to cancel the aftereffect is the highest for the physically separated test pattern and next highest for the valid luminance pattern. For one subject, the required saturation is the highest for the valid luminance patterns and the next highest for the physically separated test pattern.

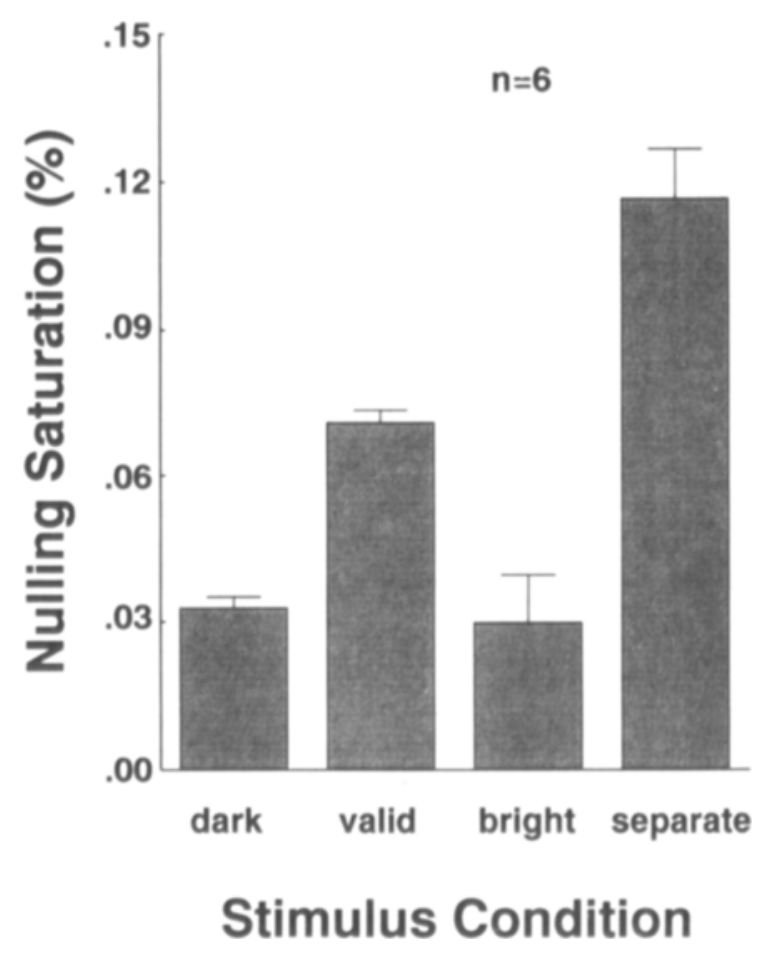

Figure 3. The mean saturation required to null the color aftereffect (expressed as excitation purity) as a function of the test stimulus conditions (too-dark, valid, too-bright, and physically separated conditions). Vertical bars represent standard errors. 
The data were subjected to a one-way (four test patterns) analysis of variance. The results showed that the pattern factor was significant $[F(3,15)=14.87, p<$ $.0001]$. A Newman-Keuls test showed a significant difference between the physically separated pattern and the valid test pattern $(p<.01)$, the too-dark pattern $(p<$ $.01)$, and the too-bright pattern $(p<.01)$ and a significant difference between the valid test pattern and both the too-dark pattern $(p<.05)$ and the too-bright pattern $(p<.05)$.

Results of $t$ tests showed that the measured mean saturation needed to cancel the aftereffect is significantly above zero in the too-dark condition $[t(5)=5.27$, $p<.05]$, the valid luminance condition $[t(5)=5.90$, $p<.05]$, and the physically separated condition $[t(5)=$ $10.25, p<.01]$, but not in the too-bright condition $[t(5)=1.63]$.

In the pretest, we had measured the saturation for color cancellation for each test pattern before the subjects were exposed to the adaptation stimuli. For all 6 subjects, the saturation necessary to cancel color is very low compared with that measured following adaptation, and no systematic difference was found between the four test patterns. The mean saturation (expressed again as \pm standard deviation) for the valid luminance pattern, the too-dark pattern, the too-bright pattern, and the physically separated pattern were $0.001( \pm 0.001), 0.000( \pm 0.001), 0.002$ $( \pm 0.001)$, and $-0.001( \pm 0.000)$. This result indicates that no subjects had any residual orientation-contingent color aftereffect prior to the experiment.

\section{DISCUSSION}

In the valid transparency pattern, in which all the observers reported seeing vertical and horizontal structure, the amount of red and green added to the test stimulus to null the aftereffect was significantly higher than in the invalid (too-dark and too-bright) transparency patterns, in which the subjects more often saw a patchwork checkerboard than a vertical and horizontal structure. This result suggests that the subjective organization caused by stimuli that induce transparency (that is, the vertical and horizontal structure in the internal representation) can influence early levels of visual processing, presumably including primary visual cortex. There are, however, two alternative explanations for these results. We will consider them briefly to show that they are both ruled out by our data.
The first alternative is that the relative strength of the pattern components in the stimulus (horizontal, vertical, oblique) might influence the saturation necessary to cancel the apparent colors. Specifically, the overlapping stimuli in the experiment contain energy (contrast) not only in the horizontal and vertical directions, but also in oblique directions. The presence of these oblique components (which are absent in the isolated vertical and horizontal test stimuli) may interfere with or mask in some way the horizontal and vertical components that mediate the McCollough effect. The assumption is that the McCollough effect for vertical and horizontal edges will therefore be weaker when diagonal energy is stronger than horizontal and vertical energy in the stimulus. Table 1 shows the mean one-dimensional contrasts in the vertical (or horizontal) direction (the Michelson contrast between the mean luminances of adjacent columns, or rows, of the stimulus) and in the diagonal $\left(45^{\circ}\right)$ direction (the contrast between the mean luminances of adjacent diagonal swaths of image squares) as well as the ratio of the contrast of cardinal versus oblique components. The ratio was the highest for the too-bright luminance pattern, the second highest for the valid luminance pattern, and the lowest for the too-dark luminance pattern. Thus, this energy assumption predicts that the McCollough effect should be the strongest for the too-bright luminance pattern, the second strongest for the valid luminance pattern, and the weakest for the too-dark luminance pattern. It is clear that this prediction is not in accordance with the results shown in Figure 3, in which the McCollough effect was the strongest for the valid luminance pattern.

The second possibility is that the differences in the measured amount of saturation among these four patterns might be attributed to different thresholds of visibility of color in the four stimuli used in the experiment. In this case, it might not be the differences in the strength of the McCollough effect that are being measured, but differences in its visibility. However, the variability of the settings (the standard deviations around the mean settings) was similar for all four test patterns, suggesting that the different patterns did not differentially affect the visibility of colors in the bars as the subjects deviated from their null settings. We therefore conclude that it is the subjective organization accompanying the perception of transparency that produces the differences in strength of the McCollough effect that we observed.

Additional evidence for the influence of subjective organization on the McCollough effect comes from a study

Table 1

Contrasts of Horizontal (or Vertical) and Diagonal Luminance Variations of the Three Stimuli and Their Ratio

\begin{tabular}{lccc}
\hline & \multicolumn{3}{c}{ Stimulus } \\
\cline { 2 - 4 } \multicolumn{1}{c}{ Contrast $(\%)$} & Valid & Too Dark & Too Bright \\
\hline Horizontal (or vertical) & 42.8 & 20.0 & 55.5 \\
Diagonal & 14.3 & 66.6 & 11.1 \\
Horizontal (or vertical)/diagonal & 3.0 & 0.3 & 5.0 \\
\hline
\end{tabular}


by Uhlarik, Pringle, and Brigell (1977). They tested the aftereffect on a figure that had two possible subjective organizations-a diamond of horizontal bars on a background of vertical bars, or a set of nested Us, with each $U$ having both vertical and horizontal elements. The McCollough effect was obtained only when observers saw the diamond, that is, in the case where vertical and horizontal structures were seen to be segregated. Jenkins and Ross (1977) and Meyer and Phillips (1980) also found the McCollough effect with similar configurations. This finding, together with ours, shows that change in subjective organization can influence the McCollough effect.

Why is the McCollough effect smaller with the valid transparency pattern than with the physically separated pattern (at least, for 5 out of 6 of our subjects)? This might be because the subjective separation of transparent surfaces is not so complete as physical separation. The same tendency was found in a pattern-identification task involving overlapping digits (Watanabe \& Cavanagh, 1991, in press). The experiment measured the correct response rates of identification for overlapped digits that can be seen as subjectively transparent in terms of the valid luminance combination and for digits presented physically separately. The correct response rates for the valid transparent condition were lower than the rates for the separate condition over a considerable range of stimulus durations. That is, separation involving overlapped but transparent patterns was not as effective as physical separation.

In conclusion, the subjective organization of the vertical and horizontal stripes accompanying the perception of transparency may mediate the McCollough effect, an effect that is assumed to occur very early in information processing. However, the subjective separation is not as effective as physical separation.

\section{REFERENCES}

Beck, J., Prazdny, K., \& Ivry, R. (1984). The perception of transparency with achromatic colors. Perception \& Psychophysics, 35 , 407-422.

DE WeERT, C. M. M. (1986). Superimposition of color discrimination. Color Research \& Application, 11, 21-26.

DODWELL, C. D., \& O'SHEA, P. (1987). Global factors generate the McCollough effect. Vision Research, 27, 569-580.

Dow, B. M., \& Gouras, P. (1973). Color and spatial specificity of single units in rhesus monkey foveal striate cortex. Journal of Neurophysiology, 36, 79-100.

Hubel, D. H., \& Wiesel, T. N. (1962). Receptive fields, binocular interaction and functional architecture in the cat's visual cortex. Journal of Physiology, 160, 106-154.

Hurvich, L. M. (1981). Color vision. Sunderland: Sinauer Associates. JENKINS, B., \& Ross, J. (1977). McCollough effect depends upon perceived organization. Perception, 6, 399-400.

KERSTEN, D. (1991). Transparency and the cooperative computation of scene attributes. In M. S. Landy \& J. A. Movshon (Eds.), Computational models of visual processing (pp. 209-228). Cambridge: The MIT Press.

Livingstone, M. S., \& Hubel, D. H. (1987). Psychophysical evidence for separate channels for the perception of form, color, movement, and depth. Journal of Neuroscience, 7, 3416-3468.

MACKAY, D. M., \& MacKaY, V. (1975). Dichoptic induction of McCollough-type effects. Quarterly Journal of Experimental Psychology, 27, 225-233.

McCollough, C. (1965). Color adaptation of edge-detectors in the human visual system. Science, 149, 1115-1116.

Metelli, F. (1974). The perception of transparency. Scientific American, 230, 90-98.

Meyer, G. E., PHILlips, D. (1980). Faces, vases, subjective contours and the McCollough effect. Perception, 9, 603-606.

MURCH, G. M. (1972). Binocular relationships in a size and color orientation specific aftereffect. Journal of Experimental Psychology, 85, 241-247.

Riggs, L. A., White, K. D., \& Eimas, P. D. (1974). Establishment and decay of orientation-contingent aftereffects of color. Perception \& Psychophysics, 16, 535-542.

Skowbo, D., Timmney, F. A., Gentry, T. A., Morant, R. B (1975). McCollough effects: Experimental findings and theoretical accounts. Psychological Bulletin, 82, 497-510.

StromeYer, C. F. I. (1978). Form-color aftereffects in human vision In R. Held, H. W. Leibowitz, \& H. L. Teuber (Eds.), Handbook of sensory physiology: VIII. Perception (pp. 97-142). Berlin: SpringerVerlag.

Stromeyer, C. F. I., LANGe, A. F., \&anz, L. (1973). Spatial frequency phase effects in human vision. Vision Research, 13, 2345-2360.

Uhlarik, J., Pringle, R., \& Brigell, M. (1977). Color aftereffects contingent on perceptual organization. Perception \& Psychophysics, 22, 506-510.

Watanabe, T., \& Cavanagh, P. (1991). Transparency facilitates recognition of overlapping stimuli even for very short exposures. Investigative Ophthalmology \& Visual Science, 32, 2508.

WATANABE, T., \& CAVANAGH, P. (in press). Effect of duration of subjective transparency using the pattem identification task. Perception.

Webster, W. R., Crassini, B., \& Willenberg, K. (1987). Simultaneous color contrast from McCollough effects is spatially contingent. Perception \& Psychophysics, 41, 402-408.

White, K. D., \& RigGs, L. A. (1975). Binocular rivalry during establishment of the McCollough effect. Joumal of the Optical Society of America, 65, 1200.

\section{NOTE}

1. Although no physically transparent stimulus could have the luminance values of these two invalid figures, Figure $2 \mathrm{C}$ does not violate Metelli's laws if the dark, rather than bright, stripes are considered as the transparent surface. Beck et al. (1984) reported that transparency can be seen even in these physically unrealizable conditions (the intersections interpreted this way are too dark and require a surface that subtracts light). Our subjects did not report transparency in Figure 2C (note that the luminances in Figure 2 do not exactly reproduce the luminances of stimuli used in the experiment) perhaps because it was a more extreme violation of physical transparency than that tested by Beck et al. or perhaps because the dark surround predisposed them to take the dark areas as background.

(Manuscript received July 26, 1991 revision accepted for publication January 31, 1992.) 